\title{
LÍNEAS DE TRABAJO COMPARTIDAS EN ACREDITACIÓN EN IBEROAMÉRICA
}

\section{Nora Espí Lacomba $(*)$}

SÍNTESIS: En Iberoamérica el sector educacional, en particular el de nivel superior, constituye un espacio de interés común con amplias posibilidades para el establecimiento de mecanismos de cooperación y de integración. En mayo de 2003 quedó oficialmente constituida la Red Iberoamericana para la Acreditación de la Calidad de la Educación Superior (RIACES), como una instancia encargada de promover la cooperación y el intercambio en materia de evaluación y de acreditación de la calidad de la educación superior, y para contribuir a la garantía de la calidad de ese nivel de enseñanza en los países de la región. A continuación se comentan las diferentes líneas de trabajo compartidas en acreditación en Iberoamérica, que la creación de dicha Red han evidenciado.

SÍNTESE: Na Ibero-América o setor educacional, em particular o de nível superior, constitui um espaço de interesse comum com amplas possibilidades para o estabelecimento de mecanismos de cooperação e de integração. Em maio de 2003, foi oficialmente constituída a Rede Iberoamericana para a Acreditação da Qualidade da Educação Superior (RIACES), como uma instância encarregada de promover a cooperação e 0 intercâmbio em matéria de avaliação e de acreditação da qualidade da educação superior, e para contribuir com a garantia da qualidade desse nível de ensino nos países da região. Após, há um comentário das diferentes linhas de trabalho compartilhadas em acreditação na IberoAmérica, as quais a criação desta Rede tem evidenciado.

(*) Secretaria ejecutiva de laJ unta deAcreditación Nacional de Cuba y profesora titular de la Facultad de Química en la Universidad de La Habana. 
Los procesos de globalización iniciados a finales del pasado siglo revivieron, entre otros, los intentos por distinguir a Iberoamérica como un conjunto determinado y no como un conglomerado de países. Elementos tales como los lingüísticos (español y portugués), los religiosos, los intereses económicos, los usos y las costumbres, etc., se esgrimieron y se esgrimen como los comunes de la región, que, a la vez, la diferencian de los de otras áreas como, por ejemplo, América del Norte (Estados Unidos y Canadá).

Estas características parecen servir como puntos de partida obligados para la búsqueda de mecanismos de cooperación y de integración que beneficien a los respectivos países, y, sin lugar a dudas, el sector educacional, en particular el de nivel superior, constituye un espacio de interés común con amplias posibilidades para el establecimiento de tales mecanismos.

A pesar de las similitudes y coincidencias geográficas e históricas, se presentan obstáculos que deben ser vencidos en este espacio.

Es conocida la enorme heterogeneidad que caracteriza la realidad de los sistemas de educación superior en Iberoamérica. Más aún, dentro de dicho conjunto, los sistemas latinoamericanos de educación superior han seguido caminos propios, haciendo frente a desafíos y a realidades muy distintos a los que han determinado las transformaciones que experimenta hoy la educación superior en España y en Portugal.

Por eso, cualquier intento para poder desarrollar mecanismos de cooperación y de integración en I beroamérica, es decir, más allá de los que de forma bilateral existen y continúan desarrollándose, deberá estar encaminado a superar los mencionados obstáculos con la necesaria creatividad y objetividad para que se haga atractivo y sustentable.

Puede partirse del supuesto de que las funciones de la educación superior son las de preservar, desarrollar y promover toda la cultura de la humanidad a través de sus procesos sustantivos y en estrecho vínculo con la sociedad, llegando con ella a todos los sectores de la misma con el más amplio acceso posible. Por tanto, para poder cumplir con esas misiones y con su encargo social, las universidades deben garantizar el mejoramiento y el desarrollo continuo de las competencias, para la adaptación y la transformación de sus estructuras y de los recursos humanos ante los compromisos que establecen con la sociedad. La universidad existe, en lo fundamental, para resolver las necesidades 
educacionales que tenga en cada momento la sociedad; así demuestra su impacto y su pertinencia.

La calidad que posean las respuestas a estas demandas sociales es un tema hoy por hoy ineludible. De ahí que el desarrollo de sistemas de evaluación de la calidad en la educación superior, y la certificación pública de esta mediante los sistemas de acreditación de programas y de instituciones para dicho nivel, sean objeto de atención, de elaboración y de debate en toda agenda de discusión nacional, regional o internacional, en el propio quehacer universitario, y en la formulación en mayor o menor medida de las políticas educacionales de cualquier país. Es por eso por lo que diversos actores participan cada vez más en la elaboración, en la validación y en el fomento de tales sistemas: estudiantes, centros de educación superior, agencias de evaluación y de acreditación, sociedades de profesionales, y organismos encargados de la elaboración de políticas estatales en el campo de la educación.

Los niveles de desarrollo que en cada país se encuentran en el campo de la evaluación y de la acreditación de la calidad son también diversos. En Iberoamérica tales sistemas son más o menos recientes, y como proyectos sistemáticos han estado llevando a cabo experiencias (a excepción de Brasil, CAPES, con la acreditación del postgrado, y de Cuba, con la evaluación institucional desde 1978) que se iniciaron a principios de la década de los noventa.

Mediante la aplicación de algunos de estos sistemas de evaluación y de acreditación se acumula mayor experiencia (CONEAU en Argentina; CNAP en Chile; CNA en Colombia; COPAES en M éxico). Otros son de reciente creación, pero han comenzado con gran dinamismo sus programas ( ANECA en España; J AN en Cuba; CONEA en Ecuador; ANEAES en Paraguay, mientras otros se desarrollan en forma más integrada, como es el caso del Consejo Centroamericano de Acreditación, para apoyar el mejoramiento de la cal idad de la educación superior de los países de esta área y el desarrollo de los sistemas nacionales de la región, cuyo ejemplo lo da Costa Rica (SINAES). En países como Bolivia y Venezuela se han hecho valiosos esfuerzos, y se han efectuado numerosos estudios técnicos para la organización de sus propios sistemas de acreditación.

Si bien es desigual el desarrollo de estos sistemas, se están consolidando importantes experiencias en evaluación y acreditación, así como en la elaboración de documentos teóricos, de política, conceptuales y guías metodológicas, que pueden ser intercambiados y validados en 
diversos proyectos conjuntos, y que han contribuido a crear una cultura de la calidad en toda Iberoamérica.

Así, cuando en febrero de 2002 se hicieron los primeros intentos para constituir una red de agencias iberoamericanas destinada a la evaluación de la calidad y de la acreditación como resultado de las iniciativas de diversos países, se crearon las condiciones necesarias para que esta iniciativa fuera apoyada gradualmente por la mayoría de ellos.

Los ministros deeducación superior de los países de l beroamérica presentes en la reunión de Madrid del 27 de noviembre de 2002, expresaron su voluntad de promover y de apoyar, en sus respectivos países y en los ámbitos de sus competencias, las iniciativas y los mecanismos existentes, así como los que pudieran establecerse en el futuro en relación con la acreditación y la calidad de la educación superior.

De igual modo, manifestaron su voluntad de impulsar las iniciativas que tendieran al establecimiento de instrumentos de colaboración entre las agencias y las entidades que realizan tareas de evaluación y de acreditación de la calidad de la educación superior, de modo que pudieran actuar sobre criterios y procedimientos mutuamente reconocibles que condujeran a una consolidación efectiva de la calidad de dicho nivel de educación en sus respectivos países, y, por tanto, en el espacio iberoamericano, contribuyendo a la cohesión regional en materia de evaluación y de acreditación.

Fue así como, en mayo de 2003, quedó oficialmente constituida en Buenos Aires la Red Iberoamericana para la Acreditación de la Calidad de la Educación Superior (RIACES), una instancia encargada de promover entre los países del área la cooperación y el intercambio en materia de evaluación y de acreditación de la calidad de la educación superior, y de contribuir a la garantía de la calidad de este nivel de enseñanza en los respectivos países.

En los Estatutos de constitución quedaron resumidos los objetivos para los cuales se creaba dicha Red en los siguientes términos:

- Promover la cooperación y el intercambio de información y de experiencias entre organismos y entidades de lberoamérica cuyo objeto sea la evaluación y la acreditación de la calidad de la educación superior. 
- Facilitar la transferencia de conocimientos y de informaciones para el desarrollo de actividades que en cada país busquen el fortalecimiento y la cualificación de los procesos de evaluación y de acreditación de titulaciones o programas académicos y de instituciones de educación superior, así como de las entidades que los realizan -agencias evaluadoras y otras-, y de las organizaciones gubernamentales involucradas en la conducción de estos procesos.

- Contribuir al desarrollo progresivo de un sistema de acreditación que favorezca el reconocimiento de títulos, de períodos y de grados de estudio, así como de programas académicos y de instituciones de educación superior, para facilitar la movilidad de estudiantes, de miembros del personal académico y de profesionales, y los procesos de integración educativa regional en los países cuyos organismos y agencias de evaluación y de acreditación formen parte de la RIACES.

- Impulsar la calidad de la educación superior en los países iberoamericanos, a través de actividades propias y con el apoyo de agencias y de entidades nacionales e internacionales de cooperación.

- Apoyar la organización de sistemas, de agencias y de entidades de evaluación y de acreditación en aquellos países del área que carezcan de éstos y que manifiesten interés por dotarse de los mismos.

- Impulsar la reflexión sobre escenarios futuros de la educación superior en Iberoamérica desde la perspectiva de la evaluación y la acreditación, como instrumento de mejora permanente de la calidad de las instituciones y de los programas que imparten.

Parala consecución de estos objetivos, los países de l beroamérica integrantes de la RIACES concibieron un ambicioso plan de trabajo desde su constitución hasta finales del 2005 , que fue revisado y ampliado en la primera asamblea general de la Red, celebrada en febrero de 2004 en La Habana. El plan de trabajo de la RIACES estuvo centrado en tres objetivos: 
- El desarrollo de la propia Red.

- El fortalecimiento de las agencias.

- El desarrollo de proyectos conjuntos.

Resultó estimulante y a la vez prometedor el compromiso mostrado por los países participantes, y el empeño en transitar por los caminos de la cooperación y de la integración en materia de evaluación y de acreditación en la educación superior. En el curso de un año, desde su creación, se han logrado poner en marcha y se han desarrollado diversos proyectos, que, por su importancia, hay que destacar.

Para lograr el desarrollo de la RIACES fue imprescindible incrementar el conocimiento mutuo de los sistemas universitarios, y facilitar la identificación de las problemáticas comunes y singulares relativas a los sistemas de evaluación y de acreditación, a los marcos reguladores, etc. Varios proyectos se encaminaron al cumplimiento de este objetivo. Entre ellos, la celebración de dos seminarios (Buenos Aires, mayo de 2003, y La Habana, febrero de 2004) para la presentación y el debate de los respectivos sistemas de evaluación y de acreditación de los países, así como de las experiencias y de las perspectivas derivadas de su aplicación. A partir de estos resultados se creó un grupo coordinado por Cuba (JAN), integrado, además, por Chile (CNAP), México ( COPAES) y Nicaragua ( CNR), cuya misión fundamental, tras la realización de estudios comparados y aprovechando la información existente, serála de servir como herramienta de col aboración para la práctica y la puesta a punto de los heterogéneos sistemas que se desarrollan. La próxima asamblea general de la Red, a principios de 2005, servirá de marco para la presentación de los resultados de este proyecto.

Paralelamente, y liderado por España (ANECA), fue creado un portal de Internet que ha acogido no sólo las necesidades de difusión y de imagen de la Red, sino que poco a poco se irá convirtiendo en una plataforma ideal para cada uno de los países que la integran, y en un foro de debate para los aspectos sustanciales de la colaboración y de la integración en estas temáticas.

En el caso del fortalecimiento de las agencias en cada país, resultó evidente la necesidad de incrementar las capacidades de evaluación y de acreditación en la región, y también la de impulsar y agilizar las actividades de transferencia entre los países, con la finalidad de contri- 
buir al desarrollo articulado de los procesos de evaluación y de acreditación en la región.

Para encaminar los esfuerzos en esa dirección, se elaboró un proyecto relacionado con la formación y la capacitación en aspectos teóricos y metodológicos de la evaluación externa de programas y de instituciones de educación superior en Iberoamérica, en el que están participando casi todos los países integrantes de la RIACES y que se encuentra coordinado por Colombia ( CNA). Los objetivos del proyecto no sólo se refieren al conocimiento y a la contextualización de los diferentes model os de evaluación externa que han sido validados en los últimos años en el área, sino que se pretende acentuar la cultura de la eval uación como un proceso permanente y sistemático que contribuya al aseguramiento de la calidad de la educación superior, y que proporcionará elementos teóricos y herramientas metodológicas para la evaluación externa de programas académicos y de instituciones a profesionales y a académicos vinculados a los sistemas de educación superior de los países miembros de la RIACES.

Dicho proyecto, concebido de forma flexible, ha conllevado el diseño de distintos módulos de acuerdo con las temáticas acordadas por los países, y prevé la preparación de documentos teóricos, de foros virtuales y de talleres presenciales. Esta propuesta culminará con un ejercicio de simulación para la aplicación de los resultados alcanzados. Los temas incluidos en el proyecto abarcan desde el análisis de los modelos de evaluación y de acreditación existentes y su impacto en el mejoramiento de la calidad de la educación superior, la gestión en los procesos de evaluación y de acreditación, y los pares evaluadores y su rol en la evaluación externa, hasta la el aboración de los informes evaluativos. Esto permitirá, además, aprovechar la experiencia de aquellos países que han consolidado los respectivos sistemas evaluativos, y, por tanto, contribuirá a acelerar el proceso de creación de capacidades de evaluación y de acreditación en la región.

En general, el desarrollo de proyectos dentro de la RIACES ha sido concebido fundamentalmente con el fin de coadyuvar al mejoramiento de la calidad de los sistemas de educación superior del beroamérica, de agilizar los procesos de armonización en materia de evaluación y de acreditación, y de promover el tratamiento de los aspectos de regulación asociados a dichas materias. Avanzar en los procesos de convergencia y en la confianza mutua entre agencias resulta un imperativo en el quehacer de la RIACES. 
En cuanto a la armonización en materia de evaluación y de acreditación, el primer proyecto aprobado que se ha estado desarrollando en los últimos meses y que se encuentra en fase de terminación, ha sido la elaboración de un glosario de términos que sirva de referencia para todas las actividades de la Red, y que permita a sus integrantes la comprensión y la identificación común de los mismos. El proyecto, coordinado por España ( ANECA), ha contado con la colaboración de varios países, cuyos representantes sostuvieron recientemente intensas sesiones de trabajo en Madrid con vistas a concluir la primera versión de dicho glosario, que será sometido a la consideración de todos los miembros de la Red hasta llegar a la aprobación de su versión definitiva en la próxima asamblea general de la RIACES a principios del próximo año.

Con el objetivo de poder avanzar en los procesos de convergencia y de lograr la necesaria transparencia y confianza mutua entre las agencias, otro de los proyectos de laRIACES ha estado dirigido a establecer principios de buenas prácticas para los organismos externos de aseguramiento de la calidad. En tal sentido, se ha definido un conjunto de principios básicos sobre los cuales se ha comenzado a trabajar en ese proyecto, coordinado por México (COPAES). Entre dichos principios se distinguen la equidad y la imparcialidad en la elaboración de los dictámenes; la congruencia con su misión y con los marcos reguladores; la confiabilidad, a partir de contar con la capacidad organizativa y técnica para llevar a cabo los procesos de evaluación y de acreditación, y otros. Una vez concluido el proyecto, y sobre todo con la aplicación de los principios acordados, se podrá llegar a una consolidación efectiva del quehacer de las agencias, y a facilitar el reconocimiento mutuo de los procedimientos que se apliquen.

Tal como fue declarado en los objetivos de la RIACES, contribuir al desarrollo de los sistemas de evaluación y de acreditación debe favorecer el reconocimiento de títulos, de períodos y de grados de estudio, de programas académicos y de instituciones de educación superior, para facilitar la movilidad académica y los procesos de integración educativa regional. No obstante, si bien las denominaciones de los títulos y de las certificaciones otorgadas en los distintos países son a menudo similares en términos nominales, no siempre describen calificaciones semejantes o reflejan exigencias comparables.

El reconocimiento de títulos o de grados requiere contar con una base sustantiva que permita realizar un análisis de comparabilidad de los mismos. Por ello, para poder avanzar hacia un análisis de este tipo 
en Iberoamérica, parece indispensable contar con una especie de «mapa» de los títulos o de los grados en la región. De aquí que otro de los proyectos que la RIACES se ha comprometido a desarrollar es un estudio comparativo de títulos en Iberoamérica, mediante el cual se consiga llegar a un conjunto de términos de referencia que establezca con claridad las definiciones básicas aplicables (título, grado, certificación, habilitación, etc.) al conocimiento de las certificaciones otorgadas por las instituciones de educación superior que integran los sistemas de cada país, y, por último, poder elaborar un informe comparativo que identifique semejanzas y diferencias, y que eventualmente sugiera cursos de acción para mejorar la comparabilidad de las certificaciones en los distintos países. Este proyecto es coordinado por Chile (CNAP), ysin duda constituirá un avance significativo en el tema del reconocimiento mutuo de títulos en la región. Además, complementará los esfuerzos encaminados a lograr el reconocimiento mutuo del trabajo de las agencias de evaluación y acreditación en el área con vistas a su integración educativa.

Como puede desprenderse del resumen expuesto, en Iberoamérica son varias las líneas compartidas de la acreditación que se encuentran en fase de desarrollo. Poder disponer de una entidad coordinadora de las mismas como es la Red Iberoamericana para la Acreditación de la Calidad de la Educación Superior (RIACES), que cuenta también con el apoyo manifiesto de la mayoría de los organismos estatales encargados de definir las políticas educativas en los respectivos países, constituye un potencial para la región que deberá ser aprovechado. Por otra parte, la forma creativa y la búsqueda de variadas vías para el desarrollo de los diferentes proyectos, el interés de incluir a todos los países de Iberoamérica en este empeño, el compromiso manifiesto de sus integrantes y la conciencia de la importancia estratégica que tiene para la región el quehacer en esta esfera, son elementos que avalan la necesidad de potenciar el trabajo de la RIACES.

Sin desconocer los obstáculos que restan por vencer o los que puedan surgir, las debilidades de una organización de apenas un año de vida y las condiciones adversas en las que en ocasiones se desarrollan los sistemas de evaluación y de acreditación en algunos países del área por diversas causas, auguramos un futuro promisorio para la RIACES y un cumplimiento exitoso en las diferentes líneas de trabajo compartidas que han sido expuestas. 


\section{BIBLIOGRAFÍA}

CASTILLO, P. DEL y CRESPO, I. (2004): «Educación superior: una agenda para la cooperación entre la Unión Europea, América Latina y el Caribe», en El espacio UEALC, Madrid.

ESPí, N. (2004): «Estudios sobrelos antecedentes, situación actual yperspectivas de la evaluación y la acreditación en la República de Cuba», en La evaluación y la acreditación de la educación superior en América Latina y el Caribe, UNESCO-IESALC, La Habana, ENPSES-Mercie Group.

MORLES, V. y LEÓN, J. R. (2002): «Los estudios de postgrado en Iberoamérica yel Caribe: evolución y tendencias», en Gestión de la calidad del postgrado en Iberoamérica, Salamanca, Ediciones AUIP.

REUNIÓN DE MINISTROS DEE DUCACIÓN SUPERIOR DE I BEROAMÉRICA (2002): Declaración política de apoyo a la garantía de la calidad y la acreditación en la educación superior, Madrid.

RIACES: Acta de la I Asamblea de la Red Iberoamericana para la Acreditación de la Calidad de la Educación Superior, La Habana, 5 de febrero de 2004, Anexos 1 al 4.

- (2003): Estatutos de la Red Iberoamericana para la Acreditación de la Calidad de la Educación Superior, Buenos Aires. 


\title{
Contactar
}

Revista lberoamericana de Educación

\author{
Principal OEI
}

